\title{
CHARACTERIZATION OF NEW EXCIMER PUMPED UV LASER DYES 2. $p$-QUATERPHENYLS
}

\author{
P. A. FLEITZ and C. J. SELISKAR $\dagger$
}

Chemistry Department, University of Cincinnati, Cincinnati, Ohio 45221-0172

R. N. STEPPEL

Exciton Inc., P.O. Box 31126, Overlook Station, Dayton, Ohio 45431

\section{J. M. KAUFFMAN}

Chemistry Department, Philadelphia College of Pharmacy and Science, 43rd Street and Kingsessing Mall, Philadelphia, PA, 19104-4495

\section{J. KELLY and A. GHIORGHIS}

Chemistry Department, Massachusetts College of Pharmacy and A.H.S., 179 Longwood Avenue, Boston, MA 02115

(Received 1 March 1991; in final form 12 April 1991)

\begin{abstract}
New excimer-pumped laser dyes based on $p$-quaterphenyl are described and the associated performance parameters are presented. A discussion of the variations in the performance parameters found is made in terms of dye chemical structures. Most of the dyes studied are significantly better than the chosen reference dye BBQ. The best dye in the series (dye 3$)$ is stabilized both by ring-alkylation and by ringbridging. The net effect is the production of a new dye with a lifetime ten times greater than that of the reference dye BBQ.
\end{abstract}

KEY WORDS: $p$-quaterphenyl, laser dye, excimer pumped, ultraviolet.

\section{INTRODUCTION}

The oligophenylenes have been recognized ${ }^{1}$ as good candidates for new laser dyes in the near ultraviolet region for some time. In the case of $308 \mathrm{~nm}$ excimer pumping, many $p$-quaterphenyl dyes are attractive since their first singlet-singlet absorptions are typically in this wavelength region, thus providing for efficient pumping. However, most of the $p$-quaterphenyl derivatives are not suitable for pumping by the third harmonic of Nd:YAG $(355 \mathrm{~nm})$ since their wavelengths of maximum absorption are bathochromically shifted relative to $355 \mathrm{~nm}$. In this regard it is to be

† To whom correspondence is to be sent. 
especially noted that the ring-bridged derivatives, such as those studied here, have absorption maxima which are red-shifted relative to the parent dye $p$-quaterphenyl. Thus, many of these ring-bridged dyes are able to be pumped with $355 \mathrm{~nm} \mathrm{Nd:YAG}$ radiation providing for efficient conversion in the blue spectral region for this important pumping source. ${ }^{2}$

Selected $p$-quaterphenyl derivatives, pumped by excimer laser radiation, have been examined by Rinke, Gusten and Ache. ${ }^{3,4}$ Kauffman and co-workers ${ }^{5,6}$ have reported results for a series of $p$-quaterphenyls under flash-lamp pumping conditions. In the first paper in this series, ${ }^{7}$ we presented our work on several new derivatives of $p$-terphenyl. A preliminary account of our work on excimer laser pumped $p$-quaterphenyls has been given. ${ }^{8}$ In this paper we present a more detailed account of our results for a series of new excimer pumped $p$-quaterphenyl dyes.

\section{EXPERIMENTAL DETAILS}

The experimental apparatus and methods used in this study have been described in detail in the previous paper. ${ }^{7}$ Although these dyes have been examined over a period of months, the dye lifetime and conversion efficiency data come from measurements done under identical operating conditions. For the sake of internal standardization on a day-to-day basis, the dye BBQ, ${ }^{9}$ dye 14 in Table 1 , in $p$-dioxane solvent was used as a benchmark. Before each study the dye laser was tuned up with BBQ to give the performance values given in Table 1. In this manner, results from day-to-day, especially for lifetimes, could be related quantitatively. In this study $100 \mathrm{ml}$ volumes were used in the small Lambda Physik circulators with no cover gas circulation. The optimum concentrations listed in Table 1 are for the oscillator-preamplifier stage of the dye laser.

The dyes described in this study have been synthesized by methods previously described. ${ }^{5-8} \mathrm{BBQ}$ was used as purchased from Exciton, Inc. The solvent $p$-dioxane was purchased from Fisher Scientific Co. as an analytical reagent and used only when freshly opened.

\section{RESULTS AND DISCUSSION}

The dyes studied are identified and numbered in Table 1 where pertinent performance characteristics are also summarized. For the sake of discussion, we have separated the dyes into two classes: singly and doubly ring-bridged $p$-quaterphenyls. Using this difference in ring-bridging as a means of classification has the practical consequence of distinguishing the tuning curves of the dyes. Figures 1 and 2 demonstrate this point for several of the dyes studied. Within each bridging class, the dyes are listed in the order of increasing laser wavelength maximum. The performance characteristics of the internal standard dye BBQ and two related dyes are given last in the table. 


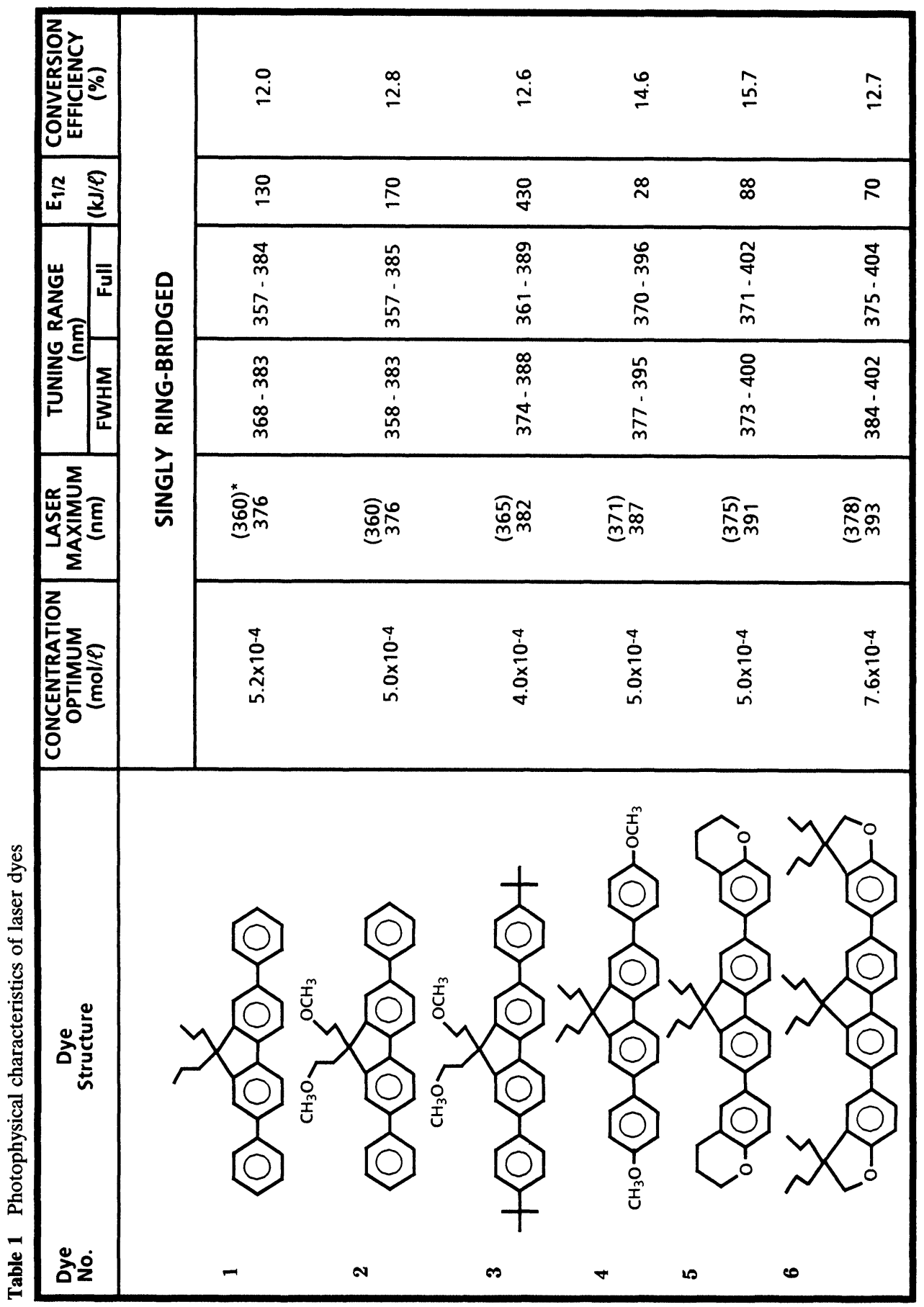




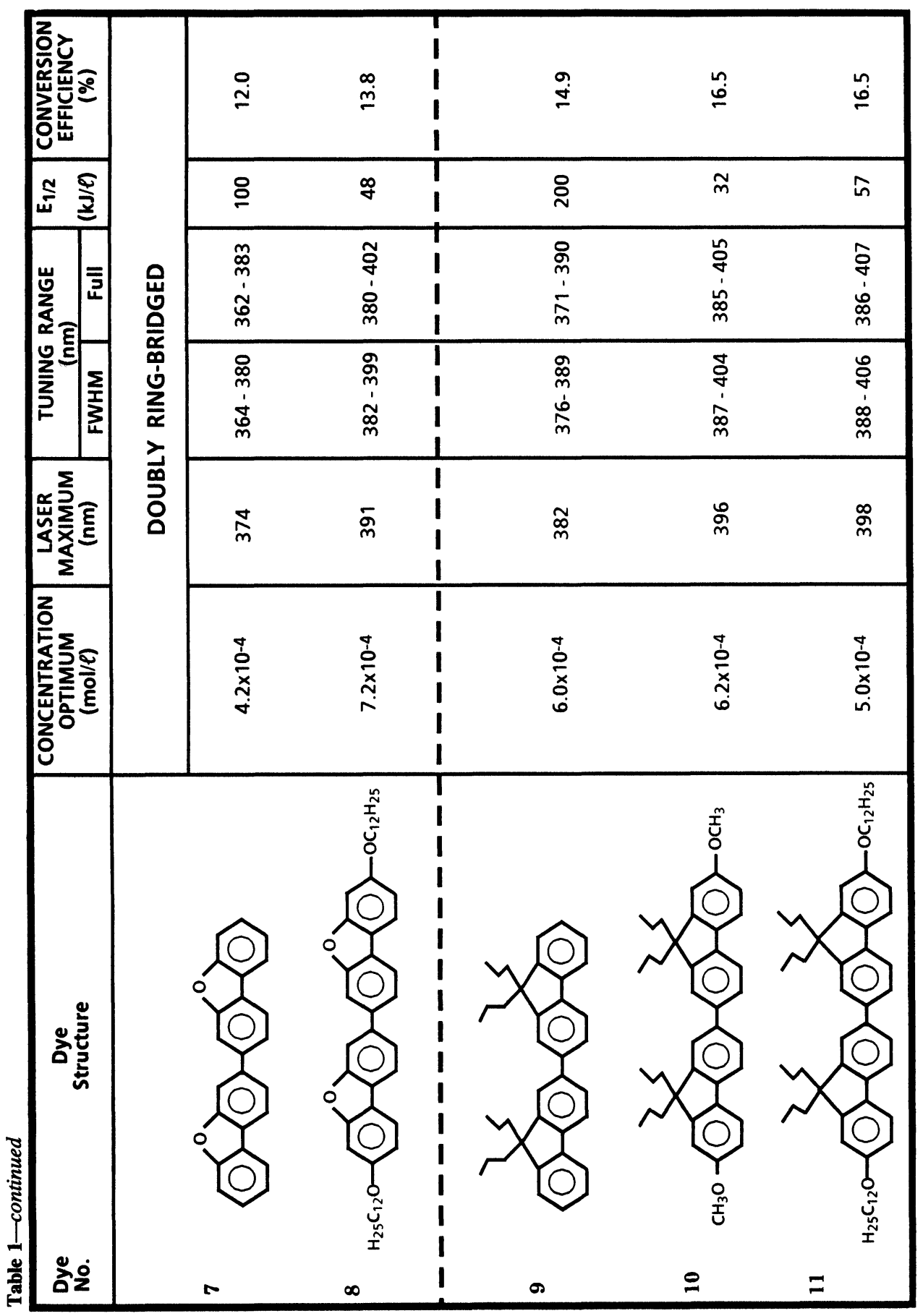




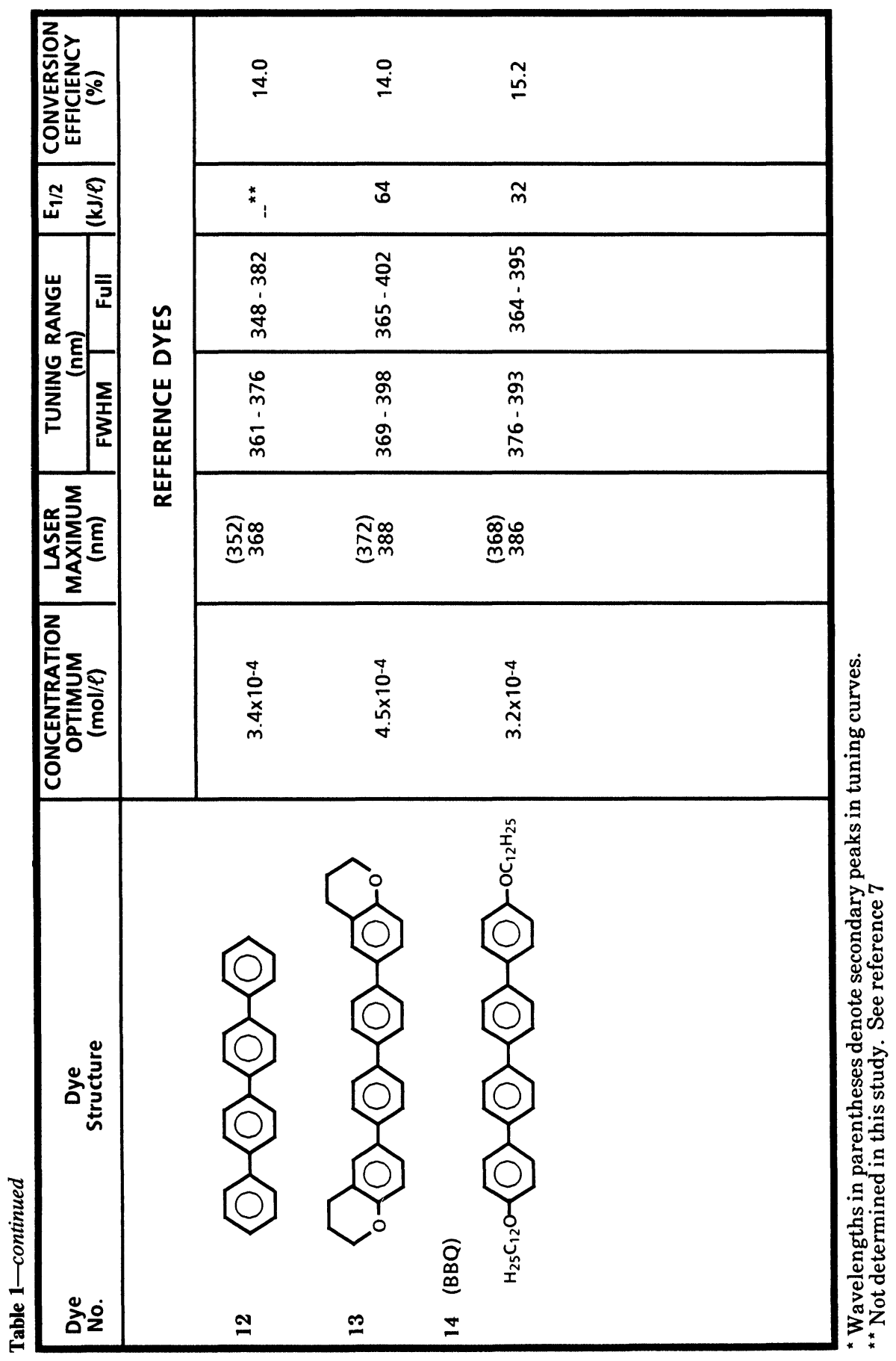




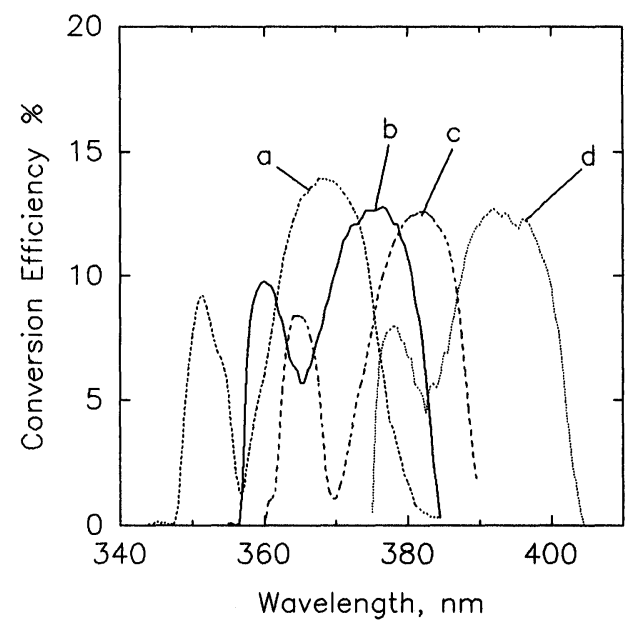

Figure 1 The tuning curves (conversion efficiency versus wavelength) of selected singly ring-bridged quaterphenyls and, for comparison, $p$-quaterphenyl, in $p$-dioxane solvent. The dye concentrations are all at the optimum values listed in Table 1 . The curves are: curve a, dye 13; curve b, dye 2; curve c, dye 3; curve d, dye 6 .

\section{General Properties of Dyes}

Overall, the conversion efficiencies listed in Table 1 vary only from 12 to $16 \%$ making any one dye acceptable from that standpoint. Compared with BBQ (dye 14), most of the lifetimes are longer and, thus, these new excimer pumped dyes represent a relative improvement in stability. Indeed, dye 3 represents more than an order of magnitude enhancement in stability relative to BBQ.

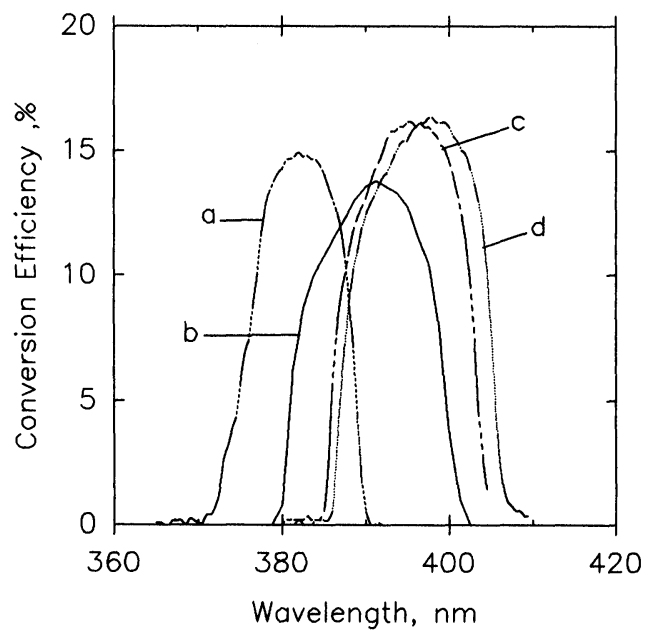

Figure 2 The tuning curves (conversion efficiency versus wavelength) of selected doubly ring-bridged quaterphenyls in $p$-dioxane solvent. The dye concentrations are all at the values listed in Table 1 . The curves are: curve a, dye 9; curve b, dye 8 ; curve c, dye 10; curve d, dye 11 . 


\section{Singly-bridged Quaterphenyls}

The singly-bridged dyes all have similarly shaped, doubly featured, tuning curves as indicated in Figure 1. In turn, these tuning curves are closely related to that of $p$-quaterphenyl itself which is also shown for the sake of comparison. In each tuning curve the shorter wavelength feature corresponds to the first vibronic component of the fluorescence; the second to the second component.

Inspection of the characteristics of dyes 1 and 2 in Table 1 in comparison with p-quaterphenyl, dye 12, shows that single ring-bridging has the effect of red-shifting the tuning curve by roughly $8 \mathrm{~nm}$. The conversion efficiencies of dyes 1 and 2 are also slightly reduced compared to dye 12 . These results are consistent with those of Rinke, Gusten and $\mathrm{Ache}^{4}$ who studied similar, but not identical, ring-bridged $p$-quaterphenyls. Substitution of methoxy groups on the ethyl side chains (dye 1 versus dye 2) gives a moderate improvement in lifetime, and only slightly affects the remaining dye parameters, as expected.

Using dyes 1 and 2 as comparison structures, it can be seen that the following conclusions hold for the remaining singly-bridged dyes:

1. The substitution of alkoxy groups at the terminal ring ends of the parent structure $($ dyes $4,5,6)$ greatly reduces the dye lifetime. In the case of the methoxy substituted dye (dye 4), the lifetime is reduced to the level of BBQ (dye 14). In this context it appears that rigidization of the aryloxy groups as found in dyes 5 and 6 , serves to partially arrest photochemical degradation. This effect is analogous to the observed increase in lifetime found in 7-amino-coumarins when the amino group is rigidized, ${ }^{10}$ reducing non-radiative loss of excited state energy. The substitution of methoxy groups (dye 4) at the terminal ring positions also results in a redshift in the tuning curve of $11 \mathrm{~nm}$. Rigidization (see dyes 5 and 6 ) further redshifts the tuning curve about $5 \mathrm{~nm}$.

2. Substitution of $t$-butyl at the terminal ring positions redshifts the tuning curve (compare dyes 2 and 3 ) by $6 \mathrm{~nm}$. This substitution also dramatically improves the dye lifetime by a factor of 2.5 . We found a very similar result for the 4,4 "- $t$-alkylsubstituted $p$-terphenyls. ${ }^{7}$ Thus, it appears that ring substitution by tertiary alkyl groups produces the most long-lived oligophenylene laser dyes.

3. While each tuning curve represents two vibronic components of the fluorescence, the usefulness of a specific dye varies due to the intensity variation in the tuning curve between the two vibronic components. For example, the wavelength region between the two components in $p$-quaterphenyl (see Figure 1), around $357 \mathrm{~nm}$, has a conversion efficiency of about $10 \%$ of the maximum value. This large "hole" in the tuning curve attenuates the usefulness of the dye in this particular region. With the exception of dye 3 , none of the singly-bridged derivatives is as extreme in this respect as $p$-quaterphenyl. Nonetheless, the "hole" in the tuning curve remains an inconvenience as one tunes through the gain profile of the dye. Almost any substitution on the basic $p$-quaterphenyl we have studied leads to an enhancement of the usefulness of the dye (see curves in Figure 1) since the minimum gain value is generally increased with chemical substitution. In a separate paper, ${ }^{11}$ we 
have also discussed how one can construct laser dye mixtures to overcome this inconvenience.

\section{Doubly-bridged Quaterphenyls}

Double ring-bridging (see dyes 7 and 9), in general, changes the shape of the tuning curves to one which has one strong broad feature as indicated in Table 1 and Figure 2. It does not matter which chemical entity, methylene or isoelectronic oxygen (see dyes 7 and 9 in Table 1), is used in the double bridging, for a single broad tuning curve results. In this regard our results are consistent with the results of Rinke, Gusten and Ache. $^{3}$

With further chemical substitution on the terminal aromatic rings, it is found that aryl-oxy derivatization (dyes 8,10 , and 11 ) results in red-shifting the tuning curve the most. Adding a complicated hydrocarbon function (dye 11 versus 10) in place of methyl on the oxygen only slightly red-shifts the tuning curve. However, such a substitution has the desirable consequence of nearly doubling the lifetime. Comparison of the parent doubly-bridged dyes (dyes 7 and 9) yields several interesting facts. First, the oxy-bridged dye is blue-shifted compared to the substituted methylene dye. Second, the stability (half-life) of the substituted methylene derivative, dye 9, is greater by a factor of 2 than the corresponding oxy-bridged compound, dye 7 . This relative stability ratio does not at first appear to be consistent with the results of Rinke, Gusten and Ache, ${ }^{3}$ who found the opposite ratio for the unsubstituted methylene-bridged dye. As has previously been discussed ${ }^{5}$ substituting all hydrogens with alkyl groups makes an important contribution to dye stability. A similar discussion may be applied to these $p$-quaterphenyl dyes. Thus, it is probable that the unsubstituted methylene bridge is the chemical site of weakness not observed when the bridge is protected by alkylation. Third, the lifetimes of the doubly-bridged compounds are all as good as or better than that of BBQ. As can be seen by comparing dyes 13 and 14 (BBQ), the addition of a rigidized ether group enhances the stability of the alkoxy derivative by roughly a factor of 2 , as it did in the singly ring-bridged dyes 5 and 6 versus 4 .

\section{CONCLUSIONS}

In conclusion, we have studied a series of excimer pumped $p$-quaterphenyl dyes which are significantly better than the chosen reference dye BBQ. As we have noted previously for the $p$-terphenyls, ${ }^{7}$ the ring-alkylated dyes stand out as the more stable derivatives. In turn, ring-bridging serves to enhance the stability of the $p$-quaterphenyls further. The addition of oxygen to the aromatic rings in the $p$-quaterphenyls, as in the case of the $p$-terphenyls, ${ }^{7}$ reduces the performance of the dyes significantly. However, the addition of oxygen to exocyclic alkyl groups can be beneficial to dye stability. The best dye in this series, dye 3, offers more than an order of magnitude improvement in stability at the expense of only a slight decrease in conversion efficiency compared to the reference dye BBQ. 
It would appear, comparing dye 9 with 1 , and dye 3 with 2 , that an outstanding new material could be made by combining double ring-bridging with $t$-alkylation, as in the new dye below. ${ }^{12}$

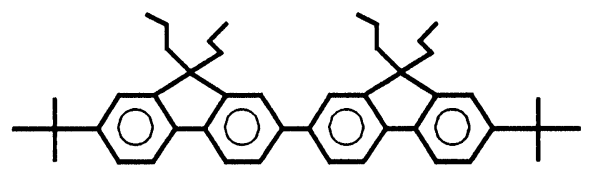

\section{Acknowledgements}

This work was supported in part by a grant to CJS and RNS from the State of Ohio Thomas Edison Fund and by the State of Ohio's Academic and Research Challenge Awards to the Chemistry Department of the University of Cincinnati. The authors thank David A. Landis and Elaine M. Seliskar for their technical assistance in preparing the manuscript. The authors thank John Montgomery of Lumonics Inc. for permission to reproduce his results for Nd:YAG pumping of these dyes. Certain of these new dyes are already available from Exciton Inc. Dyes 1-6 and 9-11 are patent pending by Exciton Inc.

\section{References}

1. K. H. Drexhage "Structure and Properties of Laser Dyes", Topics in Applied Physics, Vol. 1, Dye Lasers, F. P. Schafer, Ed., Springer-Verlag, New York (1990), pp 155-200.

2. Seven of these new dyes in $p$-dioxane solution have been evaluated by John Montgomery of Lumonics Inc. using $355 \mathrm{~nm} \mathrm{Nd:YAG} \mathrm{pumping.} \mathrm{His} \mathrm{results} \mathrm{are} \mathrm{briefly} \mathrm{summarized} \mathrm{here} \mathrm{by} \mathrm{permission} \mathrm{(see} \mathrm{Table}$ 1 for dye structures): Dye $4(389 \mathrm{~nm}, 378-395 \mathrm{~nm}, 20 \%) ;$ Dye $5(393 \mathrm{~nm}, 382-400 \mathrm{~nm}, 19 \%) ;$ Dye 7 (376 nm, 369-381 nm, 15\%); Dye $8(392 \mathrm{~nm}, 383-400 \mathrm{~nm}, 19.5 \%)$; Dye $9(384 \mathrm{~nm}, 377-388 \mathrm{~nm}$, $18 \%)$; Dye $10(396.5 \mathrm{~nm}, 388-409 \mathrm{~nm}, 20 \%)$; Dye 11 (398 nm, 391-404 nm, 21\%); where the first through third numbers in parentheses denote the lasing maximum, the full tuning range and the conversion efficiency, respectively.

3. M. Rinke, H. Gusten and H. J. Ache, J. Phys. Chem. 90, 2661-2665 (1986).

4. M. Rinke, H. Gusten and H. J. Ache, J. Phys. Chem. 90, 2666-2669 (1986).

5. J. M. Kauffman, C. J. Kelley, A. Ghiorghis, E. Neister, L. Armstrong and P. Prause, Laser Chem. 7, 343-351 (1987).

6. J. M. Kauffman, C. J. Kelley, A. Ghiorghis, E. Neister and L. Armstrong, Laser Chem. 8, 335-348 (1988).

7. D. J. Schneider, D. A. Landis, P. A. Fleitz, C. J. Seliskar, J. M. Kauffman, and R. N. Steppel, Laser Chem. 11, 49-62 (1991).

8. J. M. Kauffman, C. J. Kelley, A. Ghiorgis, E. Neister, C. J. Seliskar, and R. N. Steppel, Proc. Intl. Conf. on Lasers '89, STS Press, McLean, VA (1990), p $420 \mathrm{ff}$.

9. The dye BBQ was obtained from Exciton, Inc., Dayton, Ohio.

10. G. Jones, W. R. Jackson, C. Choi and W. R. Bergmark, J. Phys. Chem. 89, 294-300 (1985).

11. D. A. Landis, D. J. Schneider and C. J. Seliskar, Excimer Pumped Dye Mixtures for the Wavelength Region 340-380 nm, Laser Chem. xxx (1991).

12. As we showed that $t$-amyl and $t$-butyl groups were similarly effective in increasing the lifetime of $p$-terphenyl laser dyes (Ref. 7), we found it synthetically expedient to prepare 7,7'-di-t-amyl-9,9,9', $9^{\prime}$ tetraethyl-2,2'-bifluorene. The experimental conditions in these recent measurements have been refined since our measurements on the quaterphenyls listed in this paper were done. Under these refined conditions we find that the reference dye BBQ has a half-life of $67 \mathrm{~kJ} / \mathrm{liter}$ and that the new dye has a half-life of at least $500 \mathrm{~kJ} /$ liter. Thus, it is clear that the new dye is an excellent dye as predicted. 